\title{
IL-17A-Mediated Excessive Autophagy Aggravated Neuronal Ischemic Injuries via Src-PP2B-mTOR Pathway
}

\author{
Ting Liu ${ }^{1}$, Song Han ${ }^{1}$, Qingqing Dai ${ }^{1}$, Jiayin Zheng ${ }^{1}$, Cui Liu ${ }^{1}$, Shujuan $L^{2 *}$ and Junfa $L^{1 *}$ \\ ${ }^{1}$ Department of Neurobiology and Center of Stroke, Beijing Institute for Brain Disorders, School of Basic Medical Science, \\ Capital Medical University, Beijing, China, ${ }^{2}$ Department of Neurology, Beijing Chao-Yang Hospital, Capital Medical University, \\ Beijing, China
}

We previously reported that astrocyte-derived proinflammatory cytokine interleukin (IL)-17A could aggravate neuronal ischemic injuries and strength autophagy both in oxygen-glucose deprivation (OGD)/reoxygenation (R)-treated neurons and peri-infarct region of mice with middle cerebral artery occlusion (MCAO)/reperfusion (R)-simulated

\section{OPEN ACCESS}

Edited by:

Xiaoxing Xiong

Renmin Hospital, Wuhan

University, China

Reviewed by:

Enrico Castroflorio,

Medical Research Council Harwell

(MRC), United Kingdom

Mingyang Zhang

Soochow University, China

${ }^{*}$ Correspondence:

Junfa $L$

junfali@ccmu.edu.cn

Shujuan L

shujuanli@ccmu.edu.cn

Specialty section: This article was submitted to

Multiple Sclerosis and

Neuroimmunology,

a section of the journal

Frontiers in Immunology

Received: 22 September 2019 Accepted: 02 December 2019 Published: 20 December 2019

Citation:

Liu T, Han S, Dai Q, Zheng J, Liu C,

Li S and Li J (2019) IL-17A-Mediated

Excessive Autophagy Aggravated

Neuronal Ischemic Injuries via

Src-PP2B-mTOR Pathway.

Front. Immunol. 10:2952

doi: 10.3389/fimmu.2019.02952 ischemic stroke. In this study, the role and molecular mechanism of IL-17A in autophagy were further explored under ischemic condition. We found that exogenous addition of rmlL-17A remarkably $(P<0.001)$ decreased cell viability, which companying with the increases of LC3 II accumulation $(P<0.05$ or 0.01$)$ and Beclin 1 levels $(P<0.05$ or 0.001), and reduction of p62 levels $(P<0.01$ or 0.001$)$ in OGD/R-treated cortical neurons $(n=6)$. The levels of P-mTOR (Ser 2448) $(P<0.001)$ and P-S6 (Ser 240/244) $(P<0.01)$ significantly decreased without the involvement of Akt, ERK1/2 and AMPK in cortical neurons under rmIL-17A and OGD/R treatments $(n=6)$. Interestingly, the co-IP analysis exhibited that PP2B and mTOR could be reciprocally immunoprecipitated; and the addition of rmLL-17A increased their interactions, PP2B activities $(P<0.001)$, P-Src $(P<0.001)$, and P-PLC $\gamma 1(P<0.01)$ levels in OGD/R-treated neurons $(n=6$ or 5). The PP2B inhibitor Cyclosporin A blocked the induction of excessive autophagy ( $P$ $<0.05$ or $<0.001)$ and increased cell viability $(P<0.001)$ after OGD/R and rmlL-17A treatments $(n=6)$. In addition, the ICV injection of IL-17A neutralizing mAb could attenuate autophagy levels $(P<0.01$ or $0.001, n=6)$ and improve neurological functions $(P<0.01$ or $0.001, n=10)$ of mice after $1 \mathrm{~h} \mathrm{MCAO/R} 24 \mathrm{~h}$ or $7 \mathrm{~d}$. These results suggested that IL-17A-mediated excessive autophagy aggravates neuronal ischemic injuries via Src-PP2B-mTOR pathway, and IL-17A neutralization may provide a potential therapeutic effect for ischemic stroke.

Keywords: ischemic stroke, IL-17A, autophagy, OGD/R, Src-PP2B-mTOR pathway

\section{INTRODUCTION}

As the leading cause of death, ischemic stroke ( $>80 \%$ of stroke) is a medical emergency with high morbidity and mortality $(1,2)$. It causes multiple pathophysiological events including mitochondrial response, excitotoxicity, protein misfolding and immune response, which lead to the delayed neuronal loss (3). These evidences providea range of molecular mechanisms 
that are potential targets for intervention. Interleukin (IL)-17A, a potent proinflammatory cytokine, is believed to have a specific role in the delayed phase of the post-infarct inflammatory response (4). We have reported that the astrocyte-derived IL-17A reached the peak at $12 \mathrm{~h}$ or $3 \mathrm{~d}$ reperfusion ( $\mathrm{R}$ ) in peri-infarct region/cerebrospinal fluid (CSF) and serum of mice after $1 \mathrm{~h}$ middle cerebral artery occlusion (MCAO), respectively (5). In addition, the injection of IL-17A neutralizing monoclonal antibody $(\mathrm{mAb})$ could reduce the infarct volume and improve neurological outcome of mice with ischemic stroke $(6,7)$. However, how this intervention functions still remain unclear.

Autophagy is a self-protecting cellular process, through which misfolded proteins, protein aggregates, and dysfunctional organelles are degraded into metabolic constituents and recycled for maintaining cellular homeostasis. Current reports indicate that autophagy is controlled by a complicated signaling network (8). The mechanistic target of rapamycin (mTOR) is involved in the negative regulation of autophagy, and its major upstream regulators are Akt, extracellular signalregulated kinase (ERK) and adenosine 5'-monophosphateactivated protein kinase (AMPK) (9). Autophagy is closely related with inflammatory responses and the relationship between them is extraordinarily complicated (10). Following engagement of the IL-17RA/C receptor complex in neurons by IL-17A, nonreceptor tyrosine kinases could be recruited to the SEFIR domain in the receptor complex, resulting in the consecutive recruitment of downstream signaling pathways. IL-17A could induce autophagy via Janus kinase/signal transducer (JAK2/STAT3) and c-Jun N-terminal kinase (JNK) signaling pathway in human SMMC-7721 cells and osteoclast precursors (OCPs), respectively $(11,12)$. On the contrary, IL-17A inhibited autophagy via TAB2/TAB3-p38 mitogen-activated protein kinase pathways and mTOR signaling in Hepatocellular carcinoma (HCC) cells and keratinocytes $(13,14)$.

The activation of autophagy was witnessed in neurons after oxygen-glucose deprivation (OGD)/reoxygenation (R) and $\mathrm{MCAO} / \mathrm{R}$ treatments (15-17). However, the role of autophagy and IL-17A in ischemic stroke are still ambiguous. In this study, we explored the exact role and molecular mechanism of IL-17A in neuronal autophagy after ischemic stroke both in vivo and in vitro.

\section{MATERIALS AND METHODS}

All animal procedures were performed strictly in accordance with the recommendations in the guide for the care and use of Laboratory Animals of the National Institutes of Health and approved by the Experimental Animal Ethics Committee of the Capital Medical University (SCXK2016-0006).

\section{MCAO-Induced Ischemic Stroke Mouse Model}

Adult male C57 BL/6 J mice (6-8 W, 18-22 g) were purchased from the Jackson Laboratory (Bar. Harbor, ME, USA) and maintained in the Experimental Animal Center of Capital Medical University, PR China. They were housed under constant temperature $\left(23 \pm 2^{\circ} \mathrm{C}\right)$, humidity $(40-70 \%)$ and maintained on a 12-h light/dark cycle with food and water available.

Sixty-Four C57 BL/6 J mice were randomly divided into four groups as follows: Sham $(n=16), \operatorname{MCAO}(n=16), \operatorname{IgG}$ isotype $(n=16)$ and MCAO + IL-17A neutralizing monoclonal antibody (mAb, $n=16)$. After $1 \mathrm{~h} \mathrm{MCAO} / \mathrm{R} 24 \mathrm{~h}$, the mice from Sham $(n=6)$, MCAO $(n=6), \operatorname{IgG}$ isotype $(n=6)$, and $\mathrm{MCAO}+\mathrm{IL}-17 \mathrm{~A} \mathrm{mAb}(n=6)$ groups were used to examine the expressions of autophagy-related proteins by using immunoblotting. The left mice from Sham $(n=10)$, MCAO $(n$ $=10), \operatorname{IgG}$ isotype $(n=10)$, and MCAO $+\mathrm{IL}-17 \mathrm{~A} \mathrm{mAb}(n=10)$ groups after 7 days' reperfusions were designed to evaluate the neurological outcome.

The MCAO/R-induced ischemic stroke mouse model was prepared as previously described $(7,18,19)$. Mice were anesthetized with sodium pentobarbital $(60 \mathrm{mg} / \mathrm{kg})$ intraperitoneally (i.p.), and the body temperature was maintained at $36.5-37.5^{\circ} \mathrm{C}$ by using a heating pad during the surgery. Then the left common carotid artery (CCA), the left external carotid artery (ECA), and the internal carotid artery (ICA) were surgically exposed via a ventral midline incision. Next, the CCA and ECA were ligated, and the ICA was clipped by using microvascular aneurysm clips. After an arteriotomy was made in the ECA, a soft silicone coated surgical nylon monofilament suture $(0.23 \mathrm{~mm}$ in diameter; $3.0 \mathrm{~cm}$ in length, RWD Life Science, China) was gently inserted into the ICA through the ECA to occlude the middle cerebral artery (MCA, a point approximately $12.0 \mathrm{~mm}$ distal to the carotid bifurcation). After $1 \mathrm{~h}$ occlusion, the suture was carefully withdrawn to restore blood supply and the ECA was permanently ligated to prevent the incision from bleeding. Finally, reperfusion was achieved by loosening the temporary ligation on the CCA. Post-operative mice were placed in a temperature controlled cage with regular observation for $24 \mathrm{~h}$. Laser Doppler flowmetry (Perimed PeriFlux system 5000, Jarfalla, Stockholm, Sweden) was employed to monitor cerebral blood flow (CBF) during MCAO surgery and IL-17A mAb injection and to ensure that the blood circulation was occluded completely. Regional CBF decreased by $80 \%$ in mice after MCAO and restored totally after the suture was removed $1 \mathrm{~h}$ later. In the Sham group, mice received the same procedure, without inserting the nylon monofilament to occlude the MCA.

\section{Intracerebroventricular Injection of IL-17A Neutralizing mAb}

The IL-17A neutralizing mAb (2.0 $\mu \mathrm{g}$, \#560268; Becton Dickinson, New Jersey, USA) or mouse IgG isotype (2.0 $\mu \mathrm{g}$ ) was injected into the intracerebroventricle (ICV) of mice at $3 \mathrm{~h}$ after MCAO. The ICV injection was performed as previously described (20). Briefly, the anesthetized mice (sodium pentobarbital, $70 \mathrm{mg} / \mathrm{kg}$, i.p.) were placed upon a stereotaxic frame. The cannula (28-G, inner diameter $0.18 \mathrm{~mm}$; outer diameter $0.36 \mathrm{~mm}$ ) was lowered into the right cerebral ventricle according to the following coordinates: $0.5 \mathrm{~mm}$ posterior and $1.0 \mathrm{~mm}$ lateral to bregma, and $3.2 \mathrm{~mm}$ below the skull surface. The total volume of IL-17A neutralizing mAb and IgG isotype 
are $2 \mu \mathrm{l}$. The injection was operated at the rate of $0.2 \mu \mathrm{l} / \mathrm{min}$ for 10 mins, then retained the needle for another 10 mins.

\section{Evaluation of Neurological Functions}

The neurological functions of MCAO mice after $7 \mathrm{~d}$ reperfusions were evaluated by an observer who was blinded to the experiment design. Neurobehavioral scores were measured according to the neurological disability status scale (NDSS) reported by Rodriguez et al. (21), which has 10 progressive steps from 0 (normal) to 10 (death). The detailed criteria were as follows: 0, no neurological dysfunction; 2, slight dysfunction in mobility and presence of passivity; 4, moderate neurological dysfunction; 6, more handicapped animals with more marked hypomobility, circling, tremor, jerks and/or convulsions, forelimb flexion and moderate motor incoordination; 8, respiratory distress and total incapacity to move/coordinate; and 10 represents death due to $1 \mathrm{~h} \mathrm{MCAO/R}$ $7 \mathrm{~d}$. In all cases, where criteria for the precise score were not met, the nearest appropriate number was recorded: 1, 3, 5, 7, and 9 .

Neurological deficits were evaluated on a modified scoring system suggested by Ding et al. (22) as follows: 0 , no neurological deficit; (1) Difficulty in fully extending the contralateral forelimb; (2) Failure to extend contralateral forelimb; (3) Mild circling to the contralateral side; (4) Severe circling; and (5) Falling to the contralateral side.

Corner test was examined as described by Li et al. (23). The mouse was placed into a corner of $30^{\circ}$ that was formed by moving two cardboard pieces in front of its nose. When both sides of the vibrissae were stimulated by the two boards, the mouse reared forward and upward, then turned back to face the open end. Twenty trials were performed and the laterality index was calculated using the formula (number of left turns -number of right turns)/10. Only turns involving full rearing along either board were counted.

Beam balance test was employed to evaluate motor coordination and balance. Mice were trained to traverse a horizontal beam $0.7 \mathrm{~cm}$ wide, $120 \mathrm{~cm}$ long and $40 \mathrm{~cm}$ above the floor within $15 \mathrm{~s}$, and the test was performed at $1 \mathrm{~h} \mathrm{MCAO/R}$ $7 \mathrm{~d}$. Those mice fail to pass through the rod within $15 \mathrm{~s}$ were eliminated from the experiment after 3 days of training. The score criteria were as follows: 0 , mice can't stay on the beam; 1 , mice can stay on the beam, but can't move; 2 , mice tried to pass through the beam but failed and dropped midway; 3 , mice passed through the beam with more than $50 \%$ foot slips; 4 , mice traverse the beam successfully with fewer than $50 \%$ foot slips; 5 , mice passed the beam successfully with only one foot slip; 6 , the rat traverse the beam without foot slips.

To evaluate motor coordination and balance, rotarod test from 4 to $40 \mathrm{rpm}$ over a time course of $5 \mathrm{~min}$ was operated at $1 \mathrm{~h} \mathrm{MCAO/R} \mathrm{7d.} \mathrm{In} \mathrm{brief,} \mathrm{mice} \mathrm{were} \mathrm{placed} \mathrm{on} \mathrm{an} \mathrm{automated}$ accelerating rotating rod (LE8200, Panlab Harvard Apparatus, USA) and their latency to fall off the rod was recorded. Preoperative training was carried out for 3 days with 3 daily trials; only those mice able to remain on the rod for $5 \mathrm{~min}$ at 40 rpm were subjected to MCAO surgery. Postoperative testing was performed at 7 days after MCAO, the mice was given 3 trials at 40 rpm per day and the average time (in seconds) spent on the rod was calculated for analysis.

\section{Primary Cortical Neuron Culture}

The newborn $24 \mathrm{~h}$ C57 BL/6 J mice were rapidly decapitated and the cerebral cortices were separated. After removing the meninges and blood vessels, the cortices were cut into pieces of $0.2 \mathrm{~cm}^{3}$. Cortical neurons were dissociated with $0.25 \%$ trypsinEDTA (25200-056, Gibco, Grand Island, USA) and seeded onto six-well plates at a density of $1 \times 10^{6}$ cells/well. Cortical neurons were cultured in Dulbecco's Modified Eagle Medium (G11995500BT, Gibco, Beijing, China), which contained 10\% horse serum (16050-122, Gibco, Grand Island, NY, USA), 10\% fetal bovine serum (10099-141, Gibco, Grand Island, USA), 1\% penicillin-streptomycin solution (15070063, Life Technologies, Carlsbad, ON, Canada), and 0.25\% L-glutamine (25030-081, Gibco, Grand Island, USA). The medium was replaced by Neurobasal Medium (21103-040, Gibco, Grand Island, USA) containing 2\% B27 supplement (17504-044, Gibco, Grand Island, USA) after $4 \mathrm{~h}$. Half of the medium was changed every $72 \mathrm{~h}$.

\section{OGD/R Model}

The $1 \mathrm{~h}$ OGD $/ 24 \mathrm{~h} \mathrm{R}$ model was employed to simulate ischemia/reperfusion injury in vitro. When the neurons were cultured for 7 days, the medium was changed to glucose-free DMEM (11966-025, Gibco, Grand Island, USA) and placed in a $37^{\circ} \mathrm{C}$ hypoxia incubator (Thermo Scientific, Marietta, $\mathrm{OH}$, USA) under hypoxic conditions $\left(2 \% \mathrm{O}_{2} / 5 \% \mathrm{CO}_{2} / 93 \% \mathrm{~N}_{2}\right)$ for $1 \mathrm{~h}$. After that, glucose-free DMEM was replaced by Neurobasal Medium, which contained 2\% B27 supplement under normoxic condition $5 \% \mathrm{CO}_{2} / 21 \% \mathrm{O}_{2} / 74 \% \mathrm{~N}_{2}$ for $24 \mathrm{~h}$ reoxygenation. Recombinant mouse (rm) IL-17A (250 ng/mL, 421-ML-025/CF, R\&D System, MN), 3-MA (5 mM, S2767, Selleck Chemicals), Bafilomycin A1 (BafA1) (100 nM, S1413, Selleck Chemicals) and CsA (100 nM, S2286, Selleck Chemicals) dissolved in $4 \mathrm{mM} \mathrm{HCL}$, $\mathrm{H}_{2} \mathrm{O}$ or DMSO, respectively, were added into the medium during $\mathrm{OGD} / \mathrm{R}$ treatment. rmIL-17A dissolved in $40 \mu \mathrm{M} \mathrm{HCL}$ was used as vehicle group.

\section{Cell Viability Assay}

To estimate the effect of rmIL-17A and autophagy inhibitors (3-MA and Baf A1) on the survival rate of primary cortical neurons after $\mathrm{OGD} / \mathrm{R}$ treatment, the cells were planted at a density of $1 \times 10^{4}$ cells/well on a 96-well plate. The cell culture and OGD model were same as above mentioned. After $1 \mathrm{~h} \mathrm{OGD} / 24 \mathrm{~h} \mathrm{R}$, Cell Titer 96 Aqueous One Solution Cell Proliferation Assay (G3580, Promega, Madison, WI, USA) was employed according to the manufacturer's instructions to detect the overall survival rate of the cells.

\section{PP2B Activity}

PP2B Cellular Activity Assay Kit (BML-AK816, Enzo Life Sciences, Farmingdale, NY, USA) was used to measure cellular PP2B phosphatase activity. After $1 \mathrm{~h} \mathrm{OGD} / 24 \mathrm{~h} \mathrm{R}$, cells planted on six-well plates were washed in ice-cold TBS (20 mM Tris, $\mathrm{pH}$ $7.2,150 \mathrm{mM} \mathrm{NaCl}$ ). Cells were dissociated in 500 ul Lysis buffer with protease inhibitors and centrifuged at $100,000 \mathrm{~g}$ for $45 \mathrm{~min}$ 
at $4^{\circ} \mathrm{C}$. To remove free phosphate, the high-speed supernatant extracts were desalted by gel filtration. The extract samples were estimated using PP2B phosphatase assay kit (BML-AK804, Enzo Life Sciences) as described in the instruction manual. PP2B activity was assessed by measuring absorbance at $620 \mathrm{~nm}$ and normalized using the controls.

\section{Immunoprecipitation Assays}

Cells were lysed in IP buffer A $(50 \mathrm{mM}$ Tris- $\mathrm{HCl}(\mathrm{pH}$ 7.5) containing $2 \mathrm{mM}$ EDTA, $2 \mathrm{mM}$ EGTA, $5 \mu \mathrm{g} / \mu \mathrm{L}$ each of leupeptin, aprotinin, pepstatin $A$ and chymostatin, $50 \mathrm{nM}$ okadaic acid, $5 \mathrm{mM}$ sodium pyrophosphate, $100 \mu \mathrm{M}$ sodium vanadate, $1 \mathrm{mM}$ DTT, $50 \mathrm{mM} \mathrm{KF}, 5 \mathrm{M}$ iodoacetamide) and then incubated with $4 \mu \mathrm{g}$ antibody against mTOR/PP2B or IgG in a spin column from Protein G Immunoprecipitation Kit (IP50, Sigma-Aldrich, St. Louis, MO, USA) at $4^{\circ} \mathrm{C}$ overnight with constant rotation. After incubation, $30 \mu \mathrm{l}$ washed Protein G Agarose were transferred to the lysate and incubated at $4^{\circ} \mathrm{C}$ overnight with constant rotation. The immunoprecipitates was resolved in buffer [100 mM Glycine, 1.5 M Tris- $\mathrm{HCl}(\mathrm{pH} 8.0), 1 \times$ loading buffer] and centrifuged. The flow-through was collected and heated at $95^{\circ} \mathrm{C}$ for $5 \mathrm{~min}$ and proceed for immunoblotting.

\section{Immunofluorescent Staining}

After OGD treatment, neurons were fixed in $4 \%$ paraformaldehyde for $30 \mathrm{~min}$ at $25^{\circ} \mathrm{C}$, washed four times with PBS, and then blocked with buffer ( $8 \%$ goat serum + 0.2\% Triton X100 (101875278, Sigma-Aldrich, St. Louis, MO, USA) in PBS) for $1 \mathrm{~h}$ at $37^{\circ} \mathrm{C}$. Neurons were incubated with mouse anti-LC3 (SAB1305552, Sigma-Aldrich) at $4^{\circ} \mathrm{C}$ overnight. After washing, the secondary Alexa Fluor 488-conjugated goat anti-mouse IgG (Invitrogen, Carlsbad, CA, USA) was added and incubated for $2 \mathrm{~h}$ at $37^{\circ} \mathrm{C}$. Finally, cells were mounted with ProLong Gold antifade Mountant with DAPI (P36934, Life Technologies, Carlsbad, ON, Canada) and imaged using a Leica SP8 microscope with $63 \times 1.4$ Numerical Aperture (NA) oil objective lens (Leica, Wetzlar, Germany).

\section{Immunoblotting}

After $1 \mathrm{~h} \mathrm{OGD} / 24 \mathrm{~h} \mathrm{R}$, Cells in six-well plates were rinsed three times with PBS and homogenized in Buffer C $(50 \mathrm{mM}$ Tris- $\mathrm{HCl}$ (pH 7.5) containing 2 mM EDTA, 2 mM EGTA, $5 \mu \mathrm{g} / \mu \mathrm{L}$ each of leupeptin, aprotinin, pepstatin A and chymostatin, $50 \mathrm{nM}$ okadaic acid, $5 \mathrm{mM}$ sodium pyrophosphate, $100 \mu \mathrm{M}$ sodium vanadate, $1 \mathrm{mM} \mathrm{DTT}, 50 \mathrm{mM} \mathrm{KF}, 2 \%$ sodium dodecyl sulfate) and sonicated. Protein concentration was quantified via BCA protein assay kit (23225, Pierce Company, Rockford, IL 61101, USA), using albumin diluted in Buffer $\mathrm{C}$ as standard.

Samples loaded with equal amount of proteins $(30 \mu \mathrm{g})$ were electrophoresed on $8-10 \%$ sodium dodecyl sulfate polyacrylamide gel (SDS-PAGE) and transferred onto polyvinylidene difluoride (PVDF) membrane (10600021, GE Healthcare, Buckinghamshire, UK). Blocking was performed in $10 \%$ non-fat milk in Tween/Tris-buffered salt solution(TTBS, $20 \mathrm{mM}$ Tris-Cl, pH 7.5, 0.15 M NaCl, and $0.05 \%$ Tween20) for $1 \mathrm{~h}$ and membranes were incubated with primary antibodies overnight at $4^{\circ} \mathrm{C}$. Membranes were incubated in
Horseradish peroxidase-conjugated goat anti-mouse or antirabbit IgG (1:4,000, Thermo Scientific, Marietta, OH, USA) for $1 \mathrm{~h}$. Chemiluminescencent HRP substrate (90719, Millipore, Billerica, MA 01821, USA) was employed to detect the signals and proteins were visualized using Fusion-Capt Advance software on FUSION FX (Vilber Lourmat, Collégien, France). The primary antibodies used were LC3A/B (12741, Cell Signaling Technology, Danvers, MA, USA), Beclin 1(1:1,000,11306-1-AP, Proteintech, Rosemont, IL, USA), p62 (1:1,000, 5114, Cell Signaling Technology), P-mTOR (Ser 2448, 1:1,000, 5536, Cell Signaling Technology), mammalian target of rapamycin (mTOR) (1:1,000, 2983, Cell Signaling Technology), P-S6 (Ser 240/244, 1:1,000, 2215, Cell Signaling Technology), S6 Ribosomal Protein $(1: 1,000,2317$, Cell Signaling Technology), P-Akt (Thr 308,1:1,000, 9275, Cell Signaling Technology), Akt (1:1,000, 4691, Cell Signaling Technology), P-ERK (Tyr 202/Tyr 204, 1:1,000, 9101, Cell Signaling Technology), ERK (1:1,000, 9102, Cell Signaling Technology), P-AMPK (Thr 172, 1:1,000, 2535, Cell Signaling Technology), AMPK (1:1,000, 07-350, Millipore, St. Louis, MA, USA), P-Src Family (Tyr 416, 1:1,000, 6943, Cell Signaling Technology), Src (1:1,000, 2109, Cell Signaling Technology), P-PLC $\gamma 1$ (Tyr 783, 1:1,000, 2821, Cell Signaling Technology), PLC $\gamma 1$ (1:1,000, 2822, Cell Signaling Technology), $\beta$-actin (1:10,000, 60008-1-Ig, Proteintech, Rosemont, IL, USA), $\beta$-tubulin (1:20,000, 66240-1-Ig, Proteintech, Rosemont, IL, USA).

\section{Statistical Analysis}

Data were represented as mean \pm SEM. Immunoblots were quantified using Image J. GraphPad Prism version 7.0 (GraphPad Software, La Jolla, CA, USA) was used for data analysis. Statistical analysis was performed using one-way or two-way analysis of variance (ANOVA) followed by all pairwise multiple comparison procedures using Bonferroni test. $P<0.05$ was considered statistically significant. For the in vitro experiments, the $\mathrm{N}$ number represents 6 times of primary neuronal preparation under the same culture conditions; while for the in vivo experiments, the $\mathrm{N}$ number represents 6 mice under the same conditions.

\section{RESULTS}

\section{rmIL-17A Aggravates OGD/R-Induced Neuronal Ischemic Injuries Through Enhancing Autophagy Levels}

To determine the effect of IL-17A on autophagy after OGD/R treatment, the LC3 conversion (LC3 I to LC3 II), p62 and Beclin 1 protein levels were observed in $1 \mathrm{~h}$ OGD/R $24 \mathrm{~h}$-treated neurons. As shown in Figure 1, rmIL-17A could significantly increase the conversion ratio of LC3 II/total LC3 and Beclin 1 protein levels in $1 \mathrm{~h} \mathrm{OGD/R} 24 \mathrm{~h}$-treated primary cultured cortical neurons (A, B, and D) when compared with that of normoxic groups. Similarly, rmIL-17A could strengthen the degradation of p62 in cortical neurons (A and C) after $1 \mathrm{~h} \mathrm{OGD/R} 24 \mathrm{~h}$ treatment. In line with this, IL-17A increased the number of LC3 puncta in neurons after 


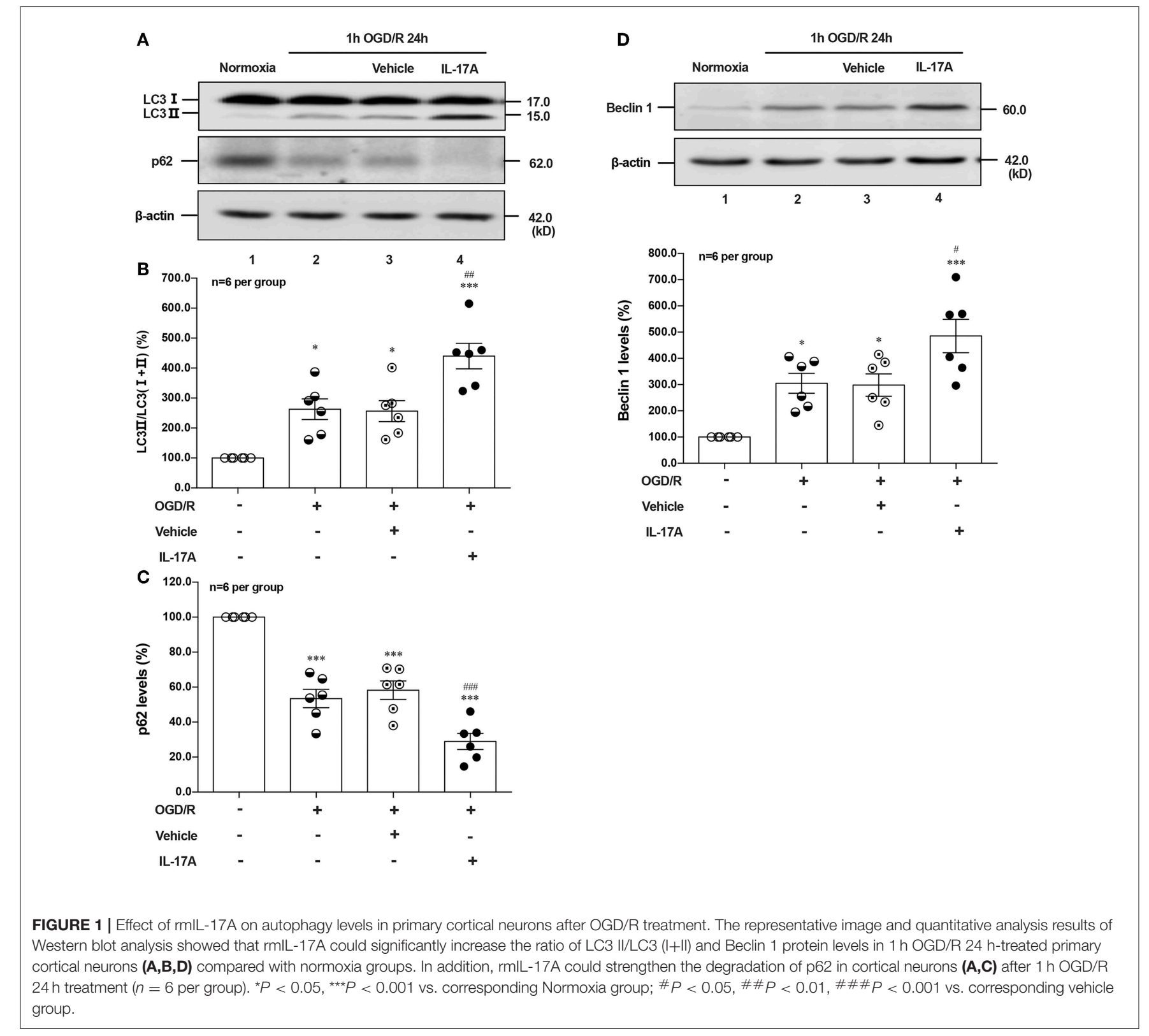

OGD treatment (Figure 5H). These results suggested that IL-17A could enhance the autophagy levels in ischemic neurons.

To further explore the role of IL-17A-enhanced autophagy levels in neuronal ischemic injuries, two autophagic inhibitors 3-methyladenine (3-MA) and Bafilomycin A1 (Baf A1) were applied. Both two inhibitors pretreatment could significantly improve the survival rates of neurons when compared with their corresponding $\mathrm{OGD} / \mathrm{R}$ or $\mathrm{OGD} / \mathrm{R}+\mathrm{IL}-$ 17A, respectively (Figure 2A). 3-MA treatment, which blocked the nucleation stage of autophagy by specifically inhibiting vacuolar protein sorting (VPS) 34 of class III phosphatidylinositol-3-kinase (PI3K) (24), could significantly decrease LC3 II accumulation in neurons exposed to OGD/R or OGD/R+IL-17A (Figure 2B). In contrast, Baf A1 could significantly increase LC3 II accumulation in neurons with
OGD/R or OGD/R+IL-17A due to the blockage of the late stages of autophagy via directly inhibiting the vacuolar $\mathrm{H}^{+}$ATPase (25) (Figure 2B). These results suggested that IL-17A aggravates neuronal ischemic injuries through enhancing the autophagy levels by modulating upstream targets of autophagy pathway.

\section{Effects of rmlL-17A on Phosphorylation Status of Positive Autophagic Pathways and Negative Regulator in OGD/R-Treated Primary Neurons}

Induction of autophagy was inhibited by interaction between ULK1 and mTOR (26), but mTOR suppression could lead to the dissociation of ULK1, thus stimulating autophagy 


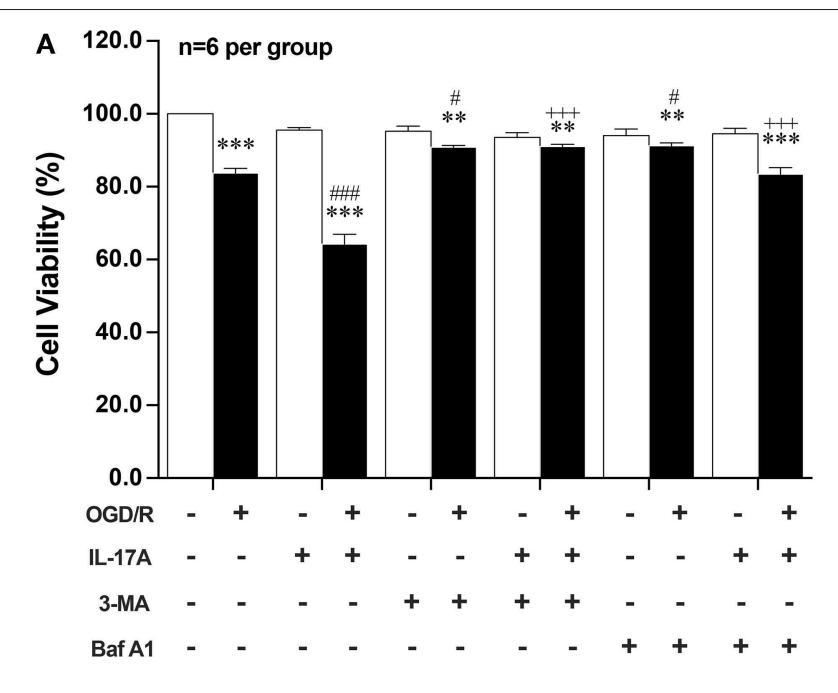

B
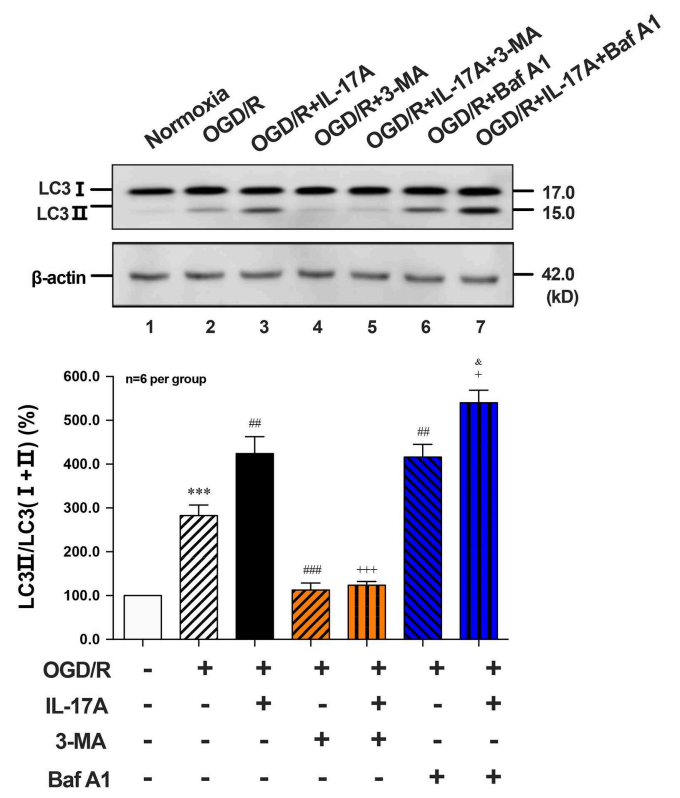

FIGURE 2 | Effects of autophagic inhibitors on cell viability and the ratio of LC3 II/LC3(I+II) in OGD/R and rmIL-17A-treated cortical neurons. The autophagic inhibitors 3-MA and Baf A1 pretreatment could significantly improve the survival rates of neurons (A) compared with their corresponding OGD/R or OGD/R+IL-17A ( $n=6$ per group). The typical and quantitative analysis results of Western blot showed that 3-MA and Baf A1treatments could significantly decrease and increase LC3 II accumulation in neurons (B) exposed to OGD/R or OGD/R+IL-17A, respectively ( $n=6$ per group). ${ }^{\star \star} P<0.01,{ }^{\star \star \star} P<0.001$ vs. corresponding Normoxia group; $\# P<0.05, \# \# P<0.01$, \#\#\# $P<0.001$ vs. corresponding OGD/R group; ${ }^{+} P<0.05,{ }^{+++} P<0.001$ vs. corresponding OGD/R+IL-17A group; \& $P<0.05$ vs. corresponding OGD/R+Baf A1 group.

(27). To determine whether autophagy was induced upon IL-17A treatment, we evaluated the phosphorylation status of $\mathrm{mTOR}$ and its downstream target $\mathrm{S} 6$ ribosomal protein. As shown in Figures 3A,B, rmIL-17A could significantly promote the decrease of P-mTOR (Ser 2448) (A) and P-S6 (Ser 240/244) (B) levels in OGD/R-treated cortical neurons, suggesting that IL-17A causes the enhanced autophagy through mTOR dephosphorylation-mediated reduction of P-S6 (Ser 240/244) level.

To explore the underlying mechanism of IL-17A-induced mTOR dephosphorylation, the effect of rmIL-17A on mTOR upstream kinases of three classic autophagy pathways were determined in OGD/R-treated neurons, including phosphorylated (P-) Akt, P-ERK1/2 and P-AMPK. As previously shown that Akt could negatively regulate AMPK, the results showed that $1 \mathrm{~h}$ OGD/R $24 \mathrm{~h}$ treatment could obviously reduce the levels of P-Akt (Figure 3C) and P-ERK1/2 (Figure 3D), but increase the P-AMPK level (Figure 3E). However, the addition of rmIL-17A did not alter the status of P-Akt, P-ERK1/2 and P-AMPK in OGD/R-treated neurons (Figures 3C-E). Thus, neither positive regulation pathway (PI3K-Akt and ERK1/2) nor negative regulator, AMPK participated in IL-17A-induced excessive autophagy during OGD/R exposure.

\section{rmIL-17A Induces Excessive Autophagy via Src-PP2B-mTOR Pathway in OGD/R Treated Neurons}

Given that PI3K-Akt, ERK1/2, and AMPK signal pathways didn't involve in IL-17A-induced dephosphorylation of mTOR during OGD/R exposure, suggesting certain type of serine (Ser)/threonine (Thr) phosphatase downstream of IL-17A signaling may be responsible. The Calcineurin/Protein Phosphatase (PP)2B is a $\mathrm{Ca}^{2+}$-associated Ser/Thr phosphatase, and has been proved physically binding to mTOR (28). In this study, the co-immunoprecipitation (cO-IP) analysis exhibited that PP2B and mTOR could reciprocally immunoprecipitated in neurons under normoxic or OGD/R conditions, and the addition of rmIL-17A could increase their interactions (Figure 4A) and PP2B activities (Figure 4B) in OGD/R-treated cortical neurons, indicating the participation of $\mathrm{PP} 2 \mathrm{~B}$ in $\mathrm{mTOR}$ dephosphorylation upon IL-17A stimulation.

As an ubiquitous Ser/Thr phosphatase, PP2B can be activated by elevated $\mathrm{Ca}^{2+}$ levels and subsequent activation of calmodulin (CaM) (29). Previous studies demonstrated that phospholipase (PL) $\mathrm{C} \gamma 1$ induced the release of $\mathrm{Ca}^{2+}$ from endoplasmic reticulum (ER) stores $(30,31)$. Given the involvement of Src protein tyrosine kinase in IL-17A signaling (32) and the interaction between PLC $\gamma 1$ and Src kinase (33), we hypothesized that IL-17A-mediated T cell receptor (TCR) signaling induced Src to activate PLC $\gamma 1$. In line with this, P-Src and P-PLC $\gamma 1$ levels were significantly increased in neurons exposed to OGD/R upon IL-17A treatment (Figures 4C,D). These results suggested that IL-17A induces the elevated autophagy via Src-PP2B-mTOR pathway in neurons exposed to OGD/R.

\section{PP2B Inhibitor CsA Suppressed rmIL-17A-Induced Excessive Autophagy and Alleviates Neuronal Injuries After OGD/R}

CsA, a potent immunosuppressant, inhibited the phosphatase activities of PP2B by competitive binding to cyclophilin A (34). The PP2B Cellular Activity Assay results confirmed 


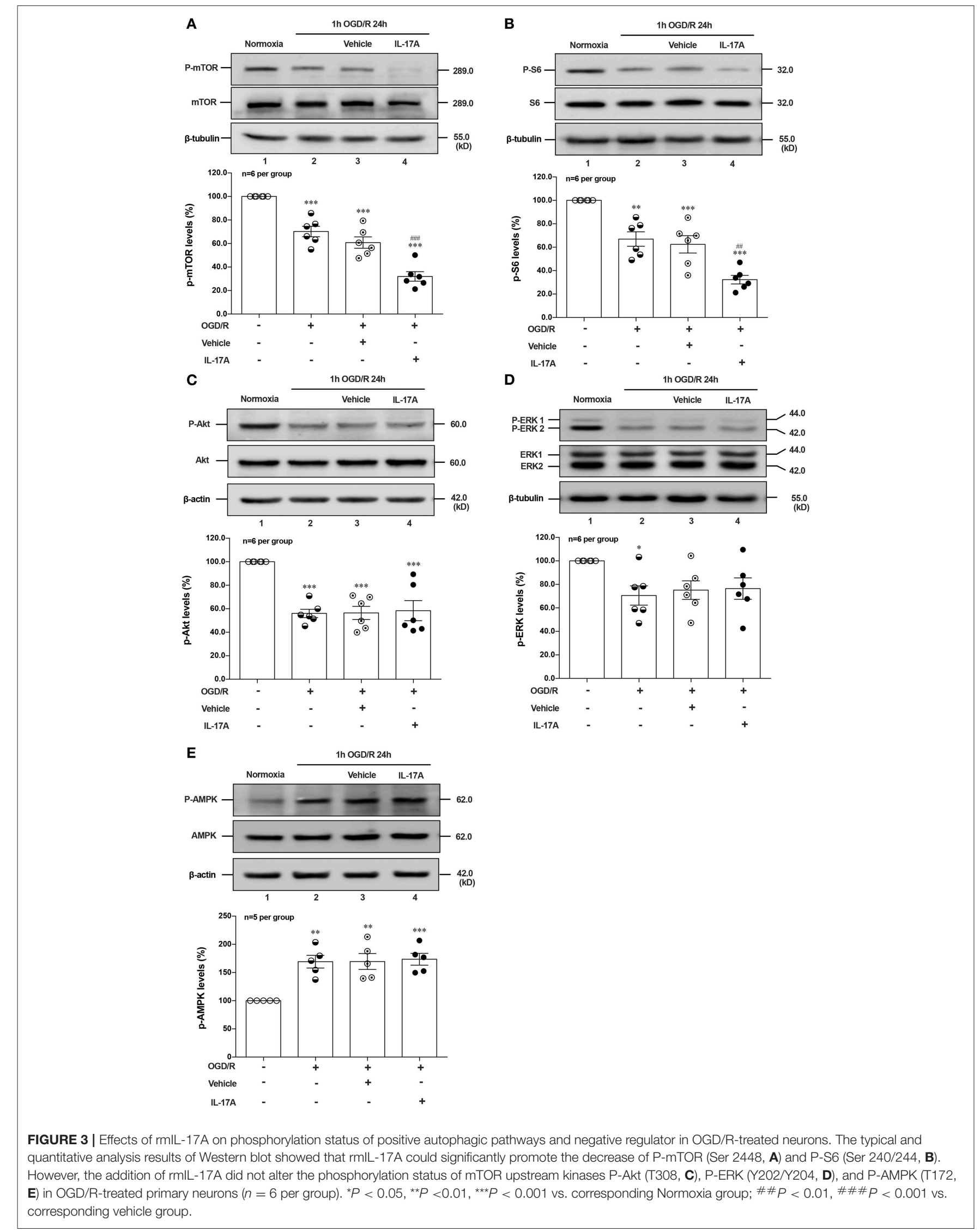


A
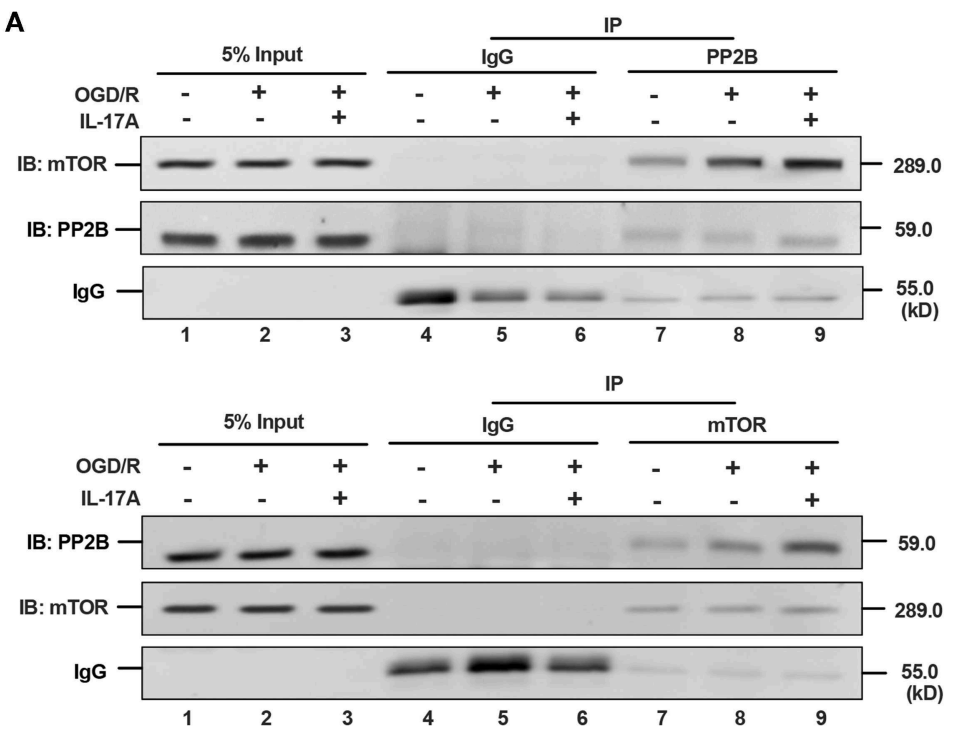

C
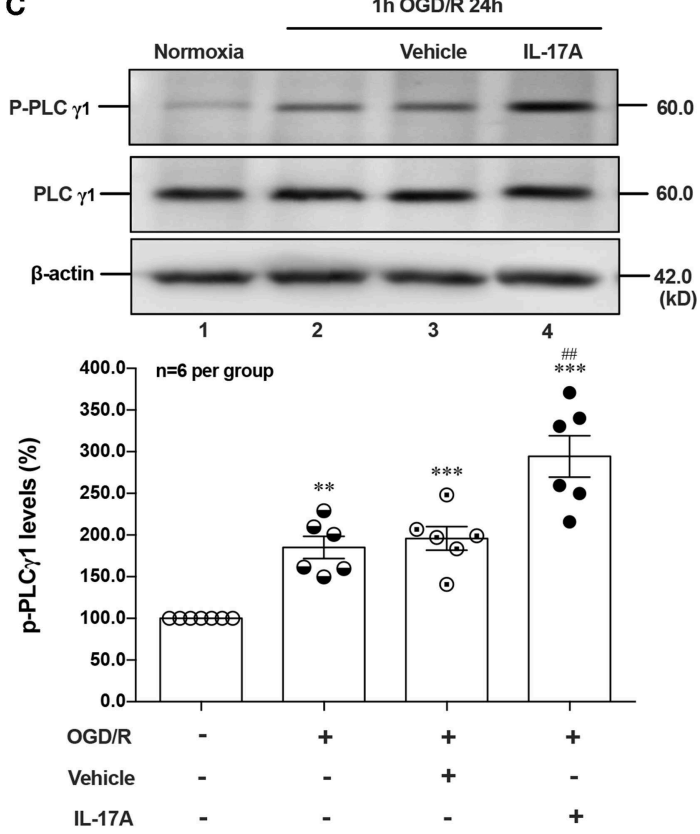

B

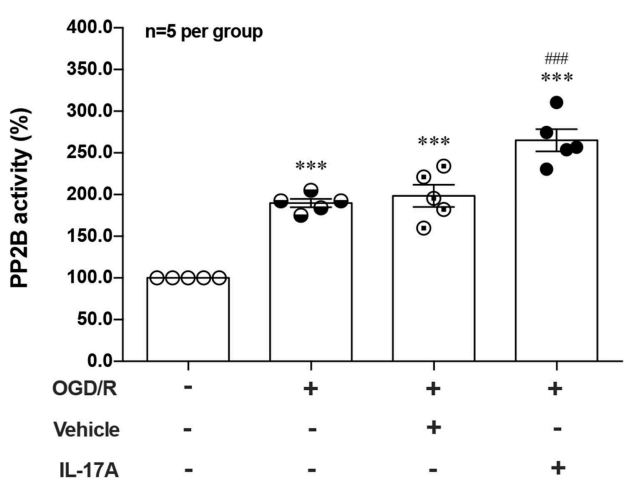

D

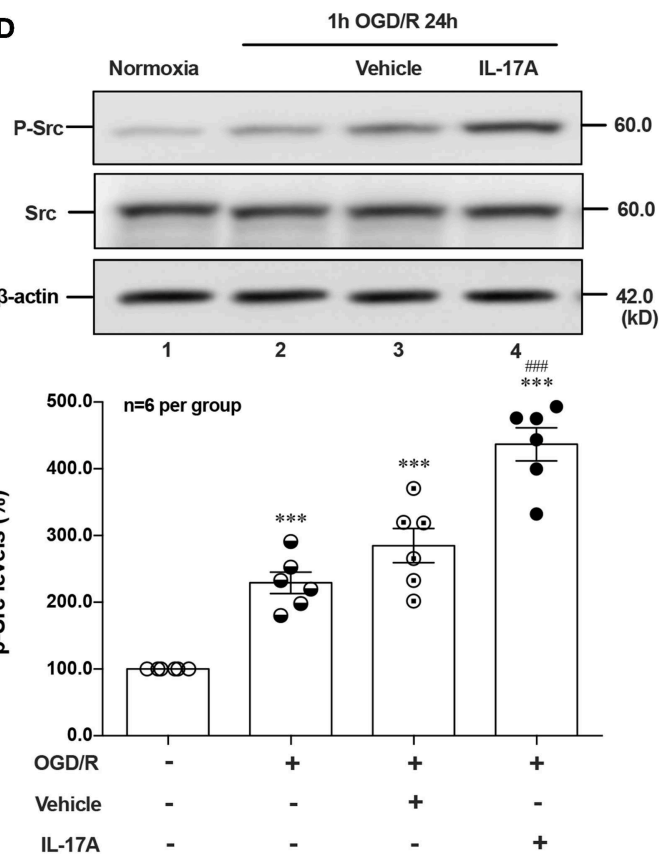

FIGURE 4 | IL-17A enhances autophagy levels via Src-PP2B-mTOR pathway in neurons exposed to OGD/R. The co-immunoprecipitation (co-IP) analysis showed that PP2B and mTOR could reciprocally immunoprecipitated in neurons under normoxic or OGD/R conditions, and the addition of rmIL-17A could increase their interactions (A) and PP2B activities (B) in OGD/R-treated cortical neurons ( $n=5$ per group). In addition, the representative and quantitative analysis results demonstrated that P-PLC $\gamma 1$ (C) and P-Src (D) levels were significantly increased in neurons exposed to OGD/R upon IL-17A treatment $\left(n=6\right.$ per group). ${ }^{\star *} P<0.01$,

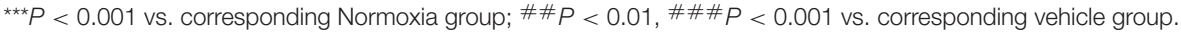

that the phosphatase activities of PP2B were obviously inhibited by CsA (Figure 5A). The P-mTOR (Ser 2448) (Figure 5B) and P-S6 (Ser 240/244) (Figure 5C) levels were restored by CsA treatment, indicating that suppression of PP2B activity reversed the dephosphorylation of mTOR at Ser 2448. To determine the effect of PP2B inactivation on the cell survival rate, the cell viability assay was applied. As shown in Figure 5D, CsA could improve neuronal cell viability after $\mathrm{OGD} / \mathrm{R}$ and $\mathrm{rmIL}-17 \mathrm{~A}$ treatments. In addition, LC3 II accumulation (Figure 5E) and Beclin 1 expression (Figure 5G) levels were decreased upon CsA treatment, meanwhile, CsA decreased the degeneration of p62 levels (Figure 5F). Consistent with this, decreased numbers of LC3 puncta can be seen upon CsA treatment. Thus, CsA suppressed IL-17A-induced excessive autophagy via PP2B inactivation. 
A

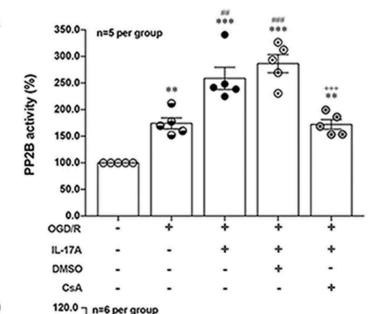

D

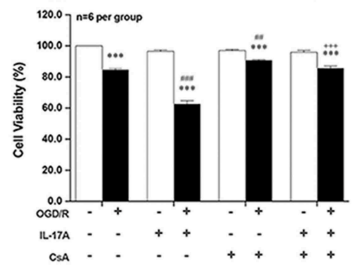

E
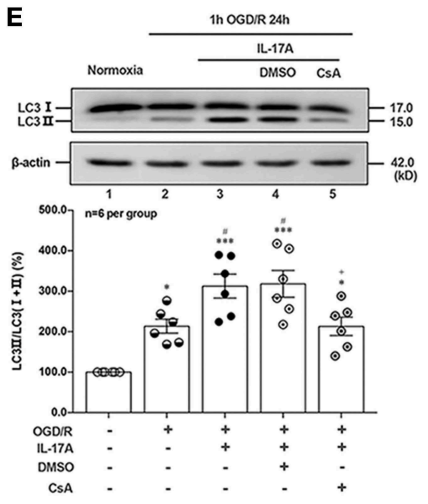

H
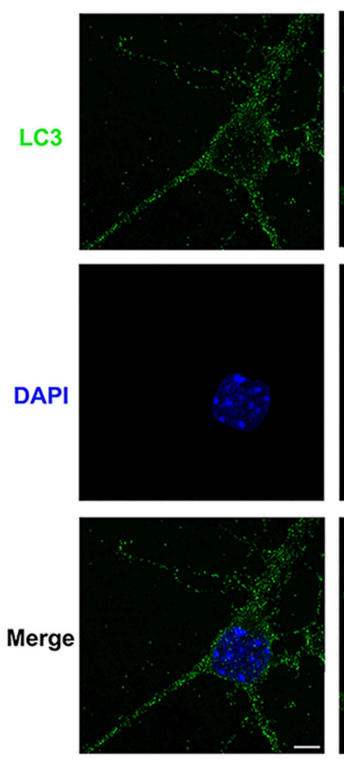

B
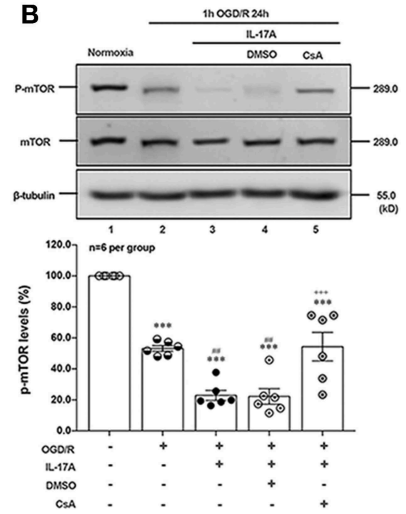

$\mathbf{F}$
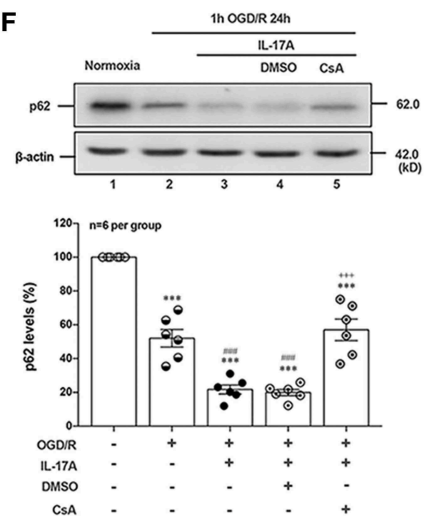

C
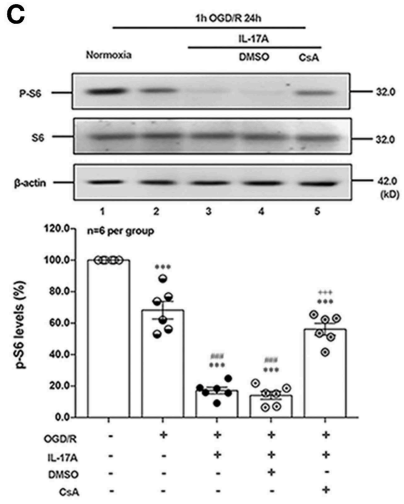
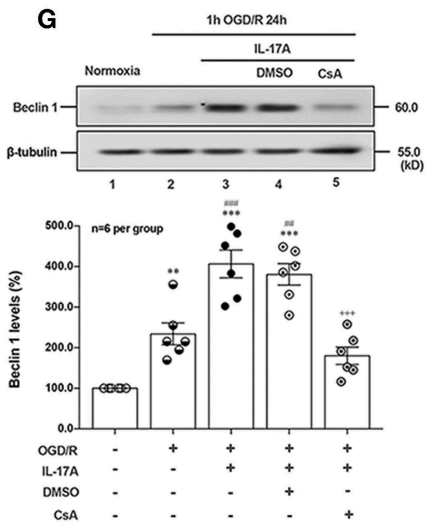

OGD

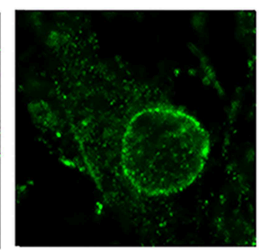

OGD+IL-17A

OGD+IL-17A+DMSO

$O G D+I L-17 A+C s A$
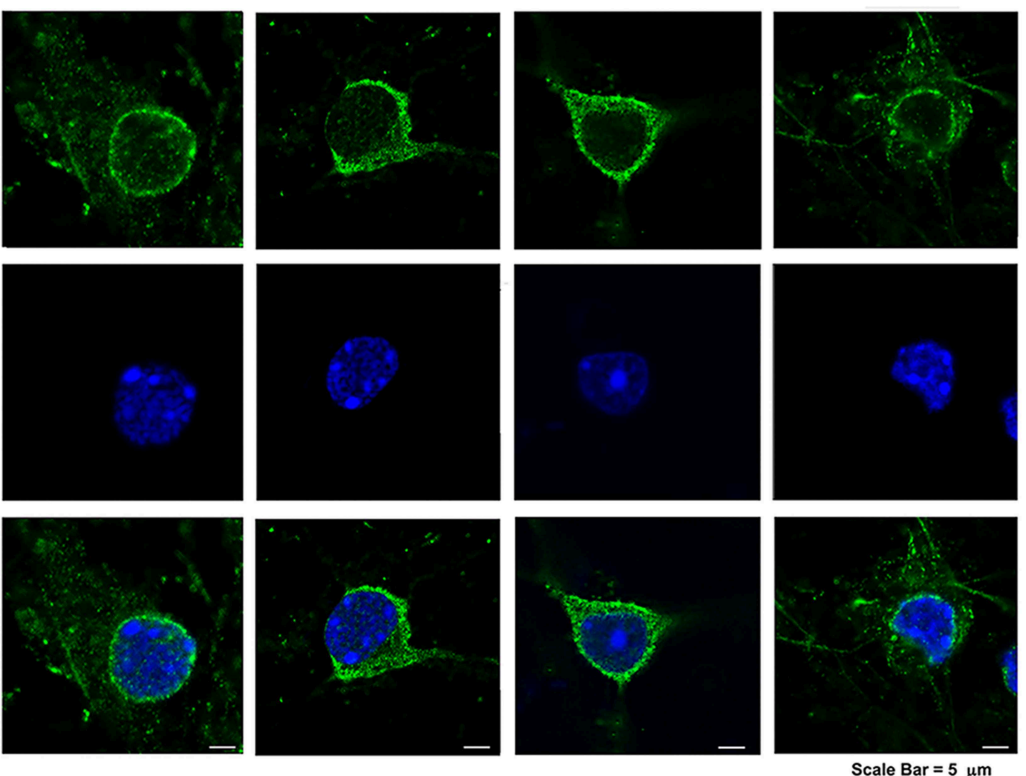

FIGURE 5 | PP2B inhibitor CsA suppresses IL-17A-induced excessive autophagy and alleviates neuronal injury in primary neurons after OGD/R. The PP2B Cellular Activity Assay results confirmed that the phosphatase activities of PP2B were obviously inhibited by CsA (A, $n=5$ per group). CsA could restore P-mTOR (Ser 2448) (B) and P-S6 (Ser 240/244) (C) levels, and improve neuronal cell viability (D) after OGD/R and rmlL-17A treatments ( $n=6$ per group). In addition, LC3 II accumulation (E), p62 degeneration (F), and Beclin 1 expression (G) levels were decreased upon CsA treatment ( $n=6$ per group). Immunofluorescent staining results showed that LC3 puncta decreased upon CsA treatment in cortical neurons $(\mathbf{H}){ }^{\star} P<0.05,{ }^{\star \star} P<0.01$, ${ }^{\star \star \star} P<0.001$ vs. corresponding Normoxia group; $\# P<0.05,{ }^{\#} \# P<0.01$, \#\#\# $P<0.001$ vs. corresponding OGD/R group; ${ }^{+} P<0.05,{ }^{+++} P<0.001$ vs. corresponding DMSO group. 


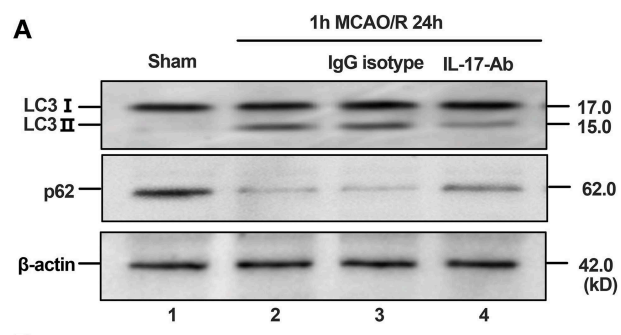

B
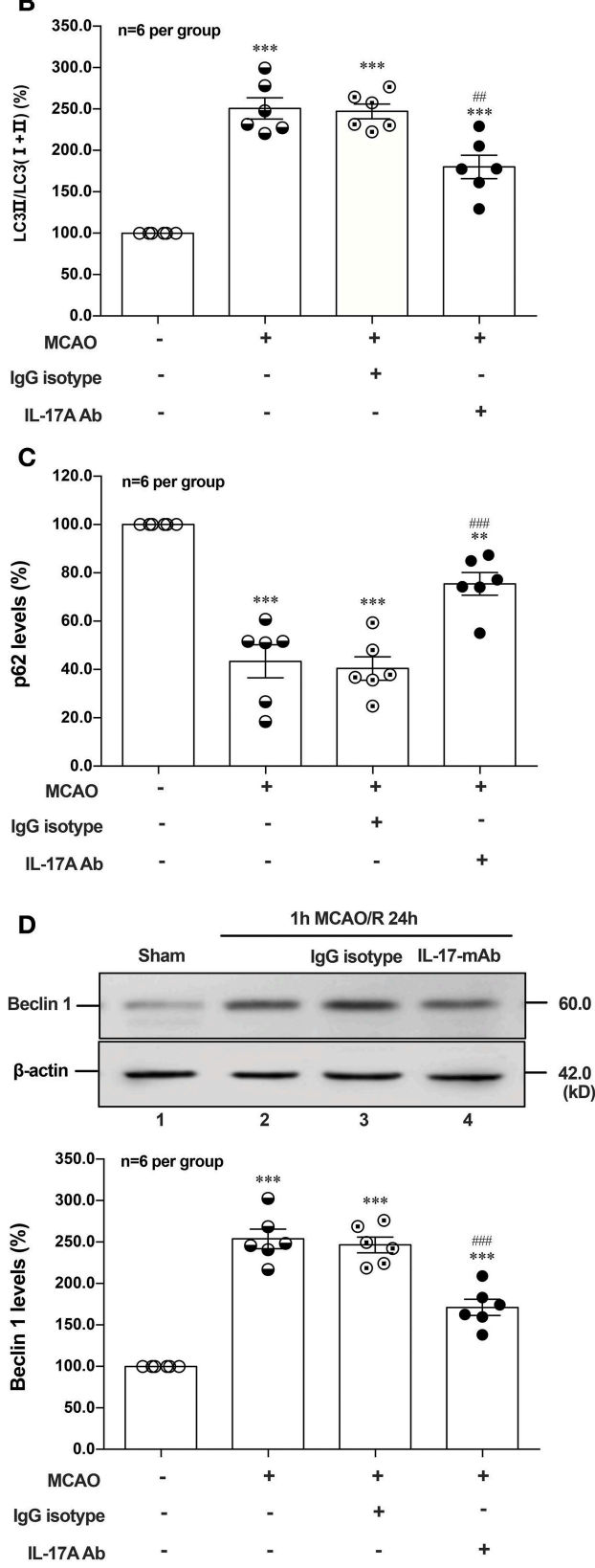

FIGURE 6 | Effect of IL-17A neutralization on autophagy levels in the peri-infarct region of mice with ischemic stroke. The representative and quantitative analysis results of Western blot showed that IL-17A neutralization could significantly decrease the conversion of LC3 I to LC3 II (A,B) and Beclin 1 protein levels (D), as well as inhibit the degradation of p62 (C)

(Continued)
FIGURE 6 | in the peri-infarct region of mice after $1 \mathrm{~h}$ MCAO/R $24 \mathrm{~h}$ ( $n=6$ per group). Data were presented as mean \pm SEM, and One-way ANOVA followed by Bonferroni test was performed. ${ }^{\star \star} P<0.01$, ${ }^{\star \star \star} P<0.001$ vs. corresponding

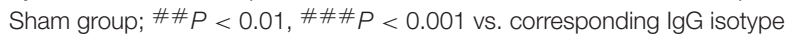
group.

\section{Neutralization of IL-17A Reduces Autophagy Levels and Improves the Neurological Outcome of Mice With Ischemic Stroke}

To determine the effect of IL-17A neutralizing $\mathrm{mAb}$ on the autophagy levels, the microtubules associated protein 1 light chain 3- $\beta$ (LC3) conversion (LC3 I-LC3 II), sequestosome (p62) and Beclin 1 protein levels were detected in the peri-infarct region of mice after $1 \mathrm{~h} \mathrm{MCAO/R} 24 \mathrm{~h}$. As shown in Figures 6A,B,D, the ratio of LC3 II/total LC3 and Beclin 1 protein levels were higher than that of Sham group, but neutralization of IL-17A could significantly decrease the accumulation of LC3 II and Beclin 1 protein levels in the peri-infarct region of mice following $1 \mathrm{~h}$ MCAO/R $24 \mathrm{~h}$. Conversely, the neutralization of IL-17A could significantly inhibit the degradation of $\mathrm{p} 62$ in the peri-infarct region of mice after $1 \mathrm{~h} \mathrm{MCAO/R} 24 \mathrm{~h}$ (Figures 6A,C). These results indicated that the autophagy levels were downregulated by neutralization of IL-17A in the peri-infarct region of mice after $1 \mathrm{~h} \mathrm{MCAO/R} 24 \mathrm{~h}$.

To explore the effect of IL-17A on the neurological outcome of ischemic stroke, IL-17A neutralizing $\mathrm{mAb}$ and mouse IgG isotype was injected into the ICV of mouse at $3 \mathrm{~h}$ after MCAO injury, after that neurological deficits and motor coordination were evaluated. Results of the neurological score (Figure 7A), the Longa score (Figure 7B), corner test (Figure 7C), beam balance test (Figure 7D), and rotarod test (Figure 7E) showed that the neurological function obviously decreased in mice after $1 \mathrm{~h} \mathrm{MCAO/R} 7 \mathrm{~d}$ when compared with that of the corresponding Sham group. However, neutralization of IL-17A could significantly improve these neurological functions of mice following $1 \mathrm{~h} \mathrm{MCAO} / \mathrm{R} 7 \mathrm{~d}$ compared with the IgG isotype group ( $n=10$ per group).

\section{DISCUSSION}

Accumulating evidence suggest that IL-17A plays a particular role in the delayed phase of the post-stroke inflammatory response $(4,6,35)$; the neutralization of IL-17A was proved to be a potential therapeutic measure for ischemic stroke (6). Our previous results also demonstrated that IL-17A levels in periinfarct cortex homogenates, CSF and serum were significantly increased in mice with ischemic stroke (5); and the blockade of IL-17A with neutralizing antibody improved the neurologic outcome of mice after ischemic stroke (7). Consistent with prior observations, we further demonstrated that IL-17A-mediated excessive autophagy aggravated neuronal ischemic injuries via Src-PP2B-mTOR pathway, and IL-17A neutralization could improve the neurological outcomes of mice with ischemic stroke. 


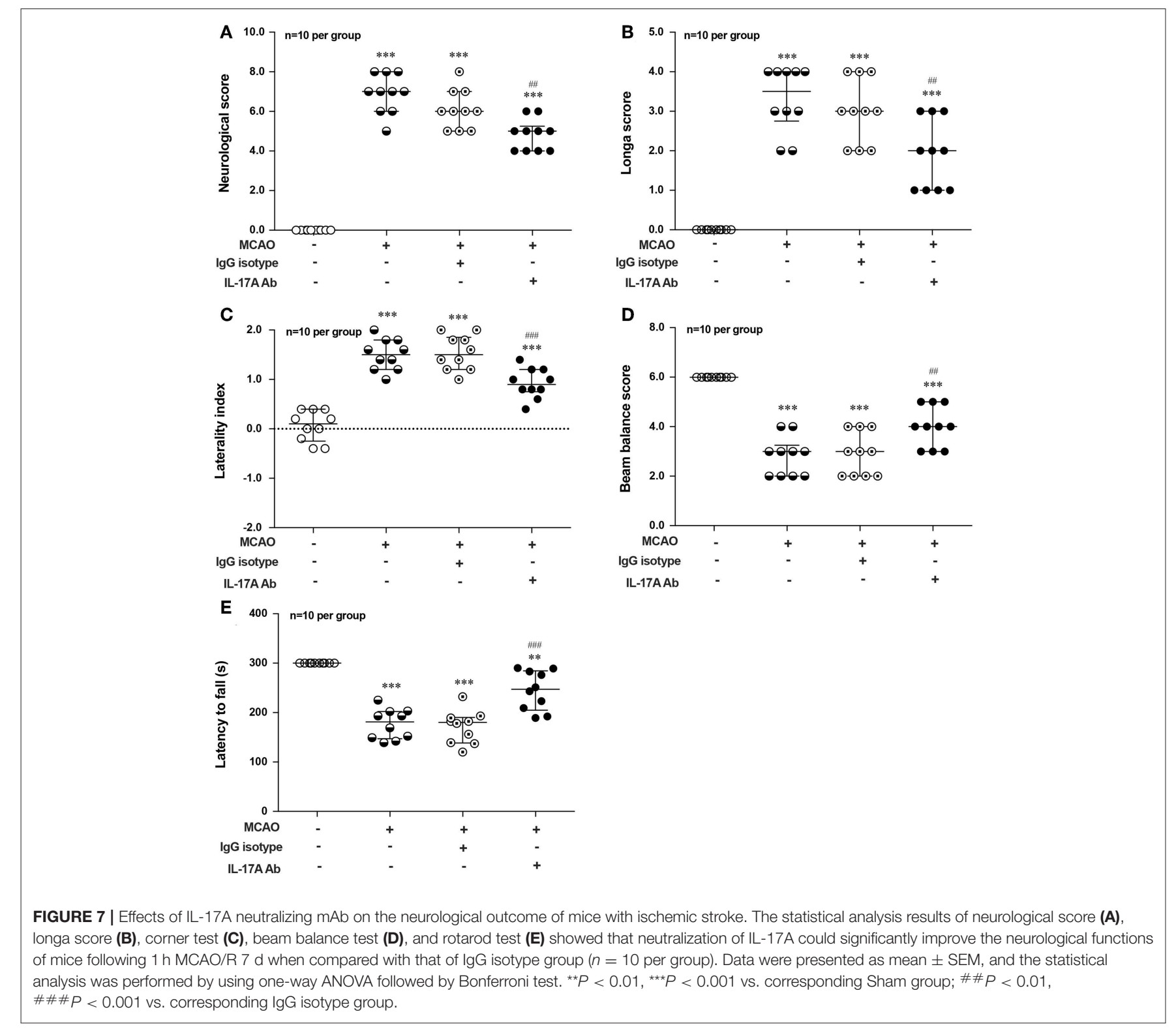

Autophagy is a double-edged sword in ischemic stroke. Basal autophagy helps cells to produce adequate energy against stressful circumstances and promotes cell survival by controlling the clearance and reuse of intracellular components. When the stress is too excessive and exceeds the maximum cellular adaptive capacity, autophagy induces cell death. Whether autophagy is beneficial or detrimental depends upon the extent of autophagy induction and the duration of autophagy activation (36). Administration of 3-MA or Baf A1 largely protected them from cell death in primary cultured cortical neurons and significantly reduced MCAO/R-induced brain infarct volume, brain edema and motor deficits, suggesting autophagy contributes to cell death both in vitro and in vivo $(16,17,37)$. In line with this, our results showed that autophagy was induced in OGD/R-treated neurons. Moreover, 3-MA and Baf A1 pre-treatment ameliorated
OGD/R-induced cell death. In contrast, neuronal autophagy upon ischemic injury could be a part of pro-survival signaling which is associated with the activation of PI3K-Akt-mTOR axis (38). These conflicting results are attributable to the variation in dosage and administration routes of pharmacological agents and different animal models.

In this study, the enhanced autophagy was observed in peri-infarct region of mice after $1 \mathrm{~h} \mathrm{MCAO/R} 24 \mathrm{~h}$; and according to our previous report that IL-17A was elevated in brain homogenates and CSF after $1 \mathrm{~h} \mathrm{MCAO} / \mathrm{R} 12 \mathrm{~h}$ (5), the role of IL-17A on neuronal autophagy was further explored after ischemic stroke. Several lines of evidence demonstrated that $\gamma \delta \mathrm{T}$ cells, T helper (Th) 17 cells as well as CNS-resident cells astrocytes and microglia, rather than neurons, are responsible for IL-17A production (39-41). 

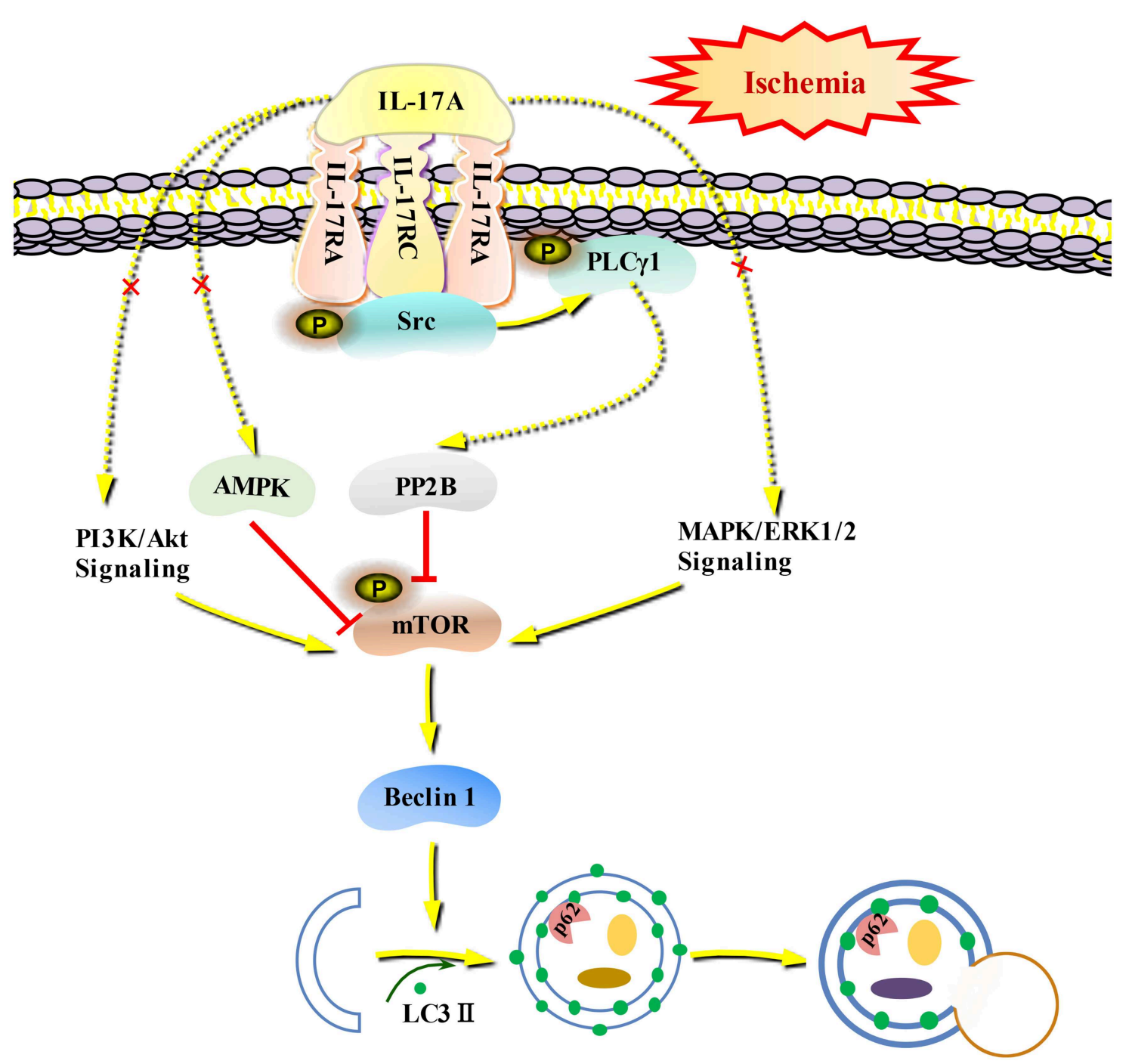

Autophagosome Autophagolysosome

FIGURE 8 | Schematic diagram of IL-17A induces excessive autophagy via Src-PP2B-mTOR pathway in neurons under ischemic condition. IL-17A stimulates IL-17A receptor-mediated signaling pathway by binding with IL-17A receptor complex IL-17RA/RC, recruiting and phosphorylating Src kinase. P-Src-mediated PLC $\gamma 1$ activation could increase PP2B activity, and then the activated PP2B induces excessive autophagy through dephosphorylating mTOR.

Thus, OGD/R treatment with the presence of IL-17A was employed to simulate in vivo ischemic stroke. We found that IL-17A aggravated OGD/R-treated ischemic injuries of primary neurons by modulating the initiation of autophagic process.

Autophagy is a self-eating cellular catabolic pathway, which is orchestrated by a complex signaling network (8). The Ser/Thr protein kinase mTOR is a key negative regulator in autophagy initiation (42). Our results showed that the phosphorylation levels of mTOR and its latter downstream target S6 were obviously decreased in primary neurons following OGD/R. Moreover, rmIL-17A treatment caused more reduction in $\mathrm{P}$ mTOR (Ser 2448) and P-S6 (Ser 240/244). Previous studies have reported that Ser 240/244 phosphorylation of S6 is regulated predominantly via an mTOR-dependent mechanism $(43,44)$. AKT/protein kinase $B(\mathrm{PKB})$ and ERK1/2, two key upstream kinases of mTOR, can positively regulate mTOR phosphorylation and thus inhibiting autophagic flux. In addition, mTOR can be inhibited by AMPK which controls intracellular energy status by sensing the AMP/ATP ratio. We found that the addition of rmIL17A didn't affect the P-Akt (T308), P-ERK1/2 (Y202/Y204), and P-AMPK (T172) in OGD/R-treated primary neurons, suggesting neither PI3K-Akt/ERK1/2 nor AMPK signaling pathways were involved in IL-17A-induced excessive autophagy during OGD/R treatment. Thus, we speculated that some type of Ser/Thr phosphatase participated in the process. It is well-known that 
PP2B plays essential roles in various processes including immune responses, nerve cell signaling and heart activity, and is the target of several therapeutic drugs that restrains the immune system (45). Previous results showed that PP2B could physically bind to mTOR via its PxIxIT motif, further proving mTOR as a direct substrate for PP2B-mediated dephosphorylation (28). Furthermore, our co-IP analysis and PP2B activity results exhibited that the addition of rmIL-17A could increase the interactions between $\mathrm{PP} 2 \mathrm{~B}$ and $\mathrm{MTOR}$ and $\mathrm{PP} 2 \mathrm{~B}$ activities in $\mathrm{OGD} / \mathrm{R}$-treated cortical neurons, indicating the participation of PP2B in mTOR dephosphorylation upon IL-17A stimulation. Elevation of intracellular calcium caused by ischemia and activation of CaM could remove an autoinhibitory helix from the active site of the phosphatase. PP2B plays a key role in activating $\mathrm{Ca}^{2+}$ signal transduction pathway. In this study, we found that IL-17A enhances autophagy level through Src-PP2B-mTOR pathway, which aggravates ischemic neuronal injury (Figure 8).

In conclusion, we firstly reported a new molecular mechanism that proinflammatory IL-17A mediated excessive autophagy to aggravate neuronal ischemic injuries via Src-PP2B-mTOR pathway. Moreover, IL-17A neutralization could improve the neurological outcomes of mice with ischemic stroke, which may provide a potential therapeutic effect for ischemic stroke in clinic.

\section{REFERENCES}

1. Waje-Andreassen U, Naess H, Thomassen L, Maroy TH, Mazengia KY, Eide $\mathrm{GE}$, et al. Biomarkers related to carotid intima-media thickness and plaques in long-term survivors of ischemic stroke. Transl Stroke Res. (2015) 6:276-83. doi: 10.1007/s12975-015-0403-0

2. Hankey GJ. Stroke. Lancet. (2017) 389:641-54. doi: 10.1016/S0140-6736(16) 30962-X

3. Zhou Z, Lu J, Liu WW, Manaenko A, Hou X, Mei Q, et al. Advances in stroke pharmacology. Pharmacol Ther. (2018) 191:23-42. doi: 10.1016/j.pharmthera.2018.05.012

4. Waisman A, Hauptmann J, Regen T. The role of IL-17 in CNS diseases. Acta Neuropathol. (2015) 129:625-37. doi: 10.1007/s00401-015-1402-7

5. Li S, Dai Q, Yu J, Liu T, Liu S, Ma L, et al. Identification of IL-17A-derived neural cell type and dynamic changes of IL-17A in serum/CSF of mice with ischemic stroke. Neurol Res. (2017) 39:552-8. doi: 10.1080/01616412.2017.1315863

6. Gelderblom M, Weymar A, Bernreuther C, Velden J, Arunachalam P, Steinbach K, et al. Neutralization of the IL-17 axis diminishes neutrophil invasion and protects from ischemic stroke. Blood. (2012) 120:3793-802. doi: 10.1182/blood-2012-02-412726

7. Dai Q, Li S, Liu T, Zheng J, Han S, Qu A, et al. Interleukin-17A-mediated alleviation of cortical astrocyte ischemic injuries affected the neurological outcome of mice with ischemic stroke. J Cell Biochem. (2019) 120:11498-509. doi: $10.1002 /$ jcb. 28429

8. Behrends C, Sowa ME, Gygi SP, Harper JW. Network organization of the human autophagy system. Nature. (2010) 466:68-76. doi: 10.1038/nature09204

9. Hou K, Xu D, Li F, Chen S, Li Y. The progress of neuronal autophagy in cerebral ischemia stroke: Mechanisms, roles and research methods. J Neurol Sci. (2019) 400:72-82. doi: 10.1016/j.jns.2019.03.015

10. Ge Y, Huang M, Yao YM. Autophagy and proinflammatory cytokines: Interactions and clinical implications. Cytokine Growth Factor Rev. (2018) 43:38-46. doi: 10.1016/j.cytogfr.2018.07.001

11. Wu J, Guo J, Cao Q, Wang Y, Chen J, Wang Z, et al. Autophagy impacts on oxaliplatin-induced hepatocarcinoma apoptosis via the

\section{DATA AVAILABILITY STATEMENT}

The datasets generated for this study are available on request to the corresponding author.

\section{ETHICS STATEMENT}

The animal study was reviewed and approved by the Experimental Animal Ethics Committee of the Capital Medical University (SCXK2016-0006).

\section{AUTHOR CONTRIBUTIONS}

TL performed the main experiments, analyzed the data and wrote the rough draft. SH, QD, JZ, and CL analyzed the data and made some of the charts. SL and JL designed the research, finalized the manuscript and received funds. All authors read and approved the final manuscript.

\section{FUNDING}

This work was supported by the National Natural Science Foundation of China grants 81771414 and 81971102 (SL), 31471142, 31671205, and 31972911 (JL).

IL-17/IL-17R-JAK2/STAT3 signaling pathway. Oncol Lett. (2017) 13:770-6. doi: $10.3892 / \mathrm{ol} .2016 .5476$

12. Ke D, Fu X, Xue Y, Wu H, Zhang Y, Chen X, et al. IL-17A regulates the autophagic activity of osteoclast precursors through RANKL-JNK1 signaling during osteoclastogenesis in vitro. Biochem Biophys Res Commun. (2018) 497:890-6. doi: 10.1016/j.bbrc.2018.02.164

13. Zhou Y, Wu PW, Yuan XW, Li J, Shi XL. Interleukin-17A inhibits cell autophagy under starvation and promotes cell migration via TAB2/TAB3-p38 mitogen-activated protein kinase pathways in hepatocellular carcinoma. Eur Rev Med Pharmacol Sci. (2016) 20:250-63.

14. Varshney P, Saini N. PI3K/AKT/mTOR activation and autophagy inhibition plays a key role in increased cholesterol during IL-17A mediated inflammatory response in psoriasis. Biochim Biophys Acta Mol Basis Dis. (2018) 1864(5 Pt A):1795-803. doi: 10.1016/j.bbadis.2018.02.003

15. Wang $\mathrm{P}$, Guan YF, Du H, Zhai QW, Su DF, Miao CY. Induction of autophagy contributes to the neuroprotection of nicotinamide phosphoribosyltransferase in cerebral ischemia. Autophagy. (2012) 8:77-87. doi: 10.4161/auto.8.1.18274

16. Zhang D, Han S, Wang S, Luo Y, Zhao L, Li J. cPKC $\gamma$-mediated downregulation of UCHL1 alleviates ischaemic neuronal injuries by decreasing autophagy via ERK-mTOR pathway. J Cell Mol Med. (2017) 21:3641-57. doi: $10.1111 /$ jcmm. 13275

17. Hua R, Han S, Zhang N, Dai Q, Liu T, Li J. cPKC $\gamma$-modulated sequential reactivation of mTOR inhibited autophagic flux in neurons exposed to oxygen glucose deprivation/reperfusion. Int J Mol Sci. (2018) 19:1380. doi: 10.3390/ijms19051380

18. Feng S, Li D, Li Y, Yang X, Han S, Li J. Insight into hypoxic preconditioning and ischemic injury through determination of nPKCepsiloninteracting proteins in mouse brain. Neurochem Int. (2013) 63:69-79. doi: 10.1016/j.neuint.2013.04.011

19. Wei H, Li Y, Han S, Liu S, Zhang N, Zhao L, et al. cPKC $\gamma$-modulated autophagy in neurons alleviates ischemic injury in brain of mice with ischemic stroke through Akt-mTOR pathway. Transl Stroke Res. (2016) 7:497-511. doi: 10.1007/s12975-016-0484-4

20. Bu X, Zhang N, Yang X, Liu Y, Du J, Liang J, et al. Proteomic analysis of cPKCßII-interacting proteins involved in HPC-induced neuroprotection 
against cerebral ischemia of mice. J Neurochem. (2011) 117:346-56. doi: 10.1111/j.1471-4159.2011.07209.x

21. Rodriguez R, Santiago-Mejia J, Gomez C, San-Juan ER. A simplified procedure for the quantitative measurement of neurological deficits after forebrain ischemia in mice. J Neurosci Methods. (2005) 147:22-8. doi: 10.1016/j.jneumeth.2005.02.013

22. Ding Y, Zhou Y, Lai Q, Li J, Park H, Diaz FG. Impaired motor activity and motor learning function in rat with middle cerebral artery occlusion. Behav Brain Res. (2002) 132:29-36. doi: 10.1016/S0166-4328(01)00405-3

23. Li X, Blizzard KK, Zeng Z, DeVries AC, Hurn PD, McCullough LD. Chronic behavioral testing after focal ischemia in the mouse: functional recovery and the effects of gender. Exp Neurol. (2004) 187:94-104. doi: 10.1016/j.expneurol.2004.01.004

24. Miller S, Oleksy A, Perisic O, Williams RL. Finding a fitting shoe for Cinderella: searching for an autophagy inhibitor. Autophagy. (2010) 6:805-7. doi: 10.4161/auto.6.6.12577

25. Clague MJ, Urbe S, Aniento F, Gruenberg J. Vacuolar ATPase activity is required for endosomal carrier vesicle formation. J Biol Chem. (1994) 269:21-4.

26. Zachari M, Ganley IG. The mammalian ULK1 complex and autophagy initiation. Essays Biochem. (2017) 61:585-96. doi: 10.1042/EBC20170021

27. Mizushima N. The role of the Atg1/ULK1 complex in autophagy regulation. Curr Opin Cell Biol. (2010) 22:132-9. doi: 10.1016/j.ceb.2009.12.004

28. Huynh $\mathrm{H}$, Wan Y. mTORC1 impedes osteoclast differentiation via calcineurin and NFATc1. Commun Biol. (2018) 1:29. doi: 10.1038/s42003-018-0028-4

29. Stewart AA, Ingebritsen TS, Manalan A, Klee CB, Cohen P. Discovery of a $\mathrm{Ca} 2+-$ and calmodulin-dependent protein phosphatase: probable identity with calcineurin. (CaM-BP80). FEBS Lett. (1982) 137:80-4. doi: 10.1016/0014-5793(82)80319-0

30. Babich A, Burkhardt JK. Coordinate control of cytoskeletal remodeling and calcium mobilization during T-cell activation. Immunol Rev. (2013) 256:8094. doi: 10.1111/imr.12123

31. Nohara LL, Stanwood SR, Omilusik KD, Jefferies WA. Tweeters, woofers and horns: the complex orchestration of calcium currents in t lymphocytes. Front Immunol. (2015) 6:234. doi: 10.3389/fimmu.2015.00234

32. Hsieh HG, Loong CC, Lin CY. Interleukin-17 induces src/MAPK cascades activation in human renal epithelial cells. Cytokine. (2002) 19:159-74. doi: 10.1006/cyto.2002.1952

33. Poulin B, Sekiya F, Rhee SG. Intramolecular interaction between phosphorylated tyrosine-783 and the C-terminal Src homology 2 domain activates phospholipase C-gamma1. Proc Natl Acad Sci USA. (2005) 102:4276-81. doi: 10.1073/pnas.0409590102

34. Liu J, Farmer JDJr, Lane WS, Friedman J, Weissman I, Schreiber SL. Calcineurin is a common target of cyclophilincyclosporin A and FKBP-FK506 complexes. Cell. (1991) 66:807-15. doi: 10.1016/0092-8674(91)90124-H

35. Zhang J, Mao X, Zhou T, Cheng X, Lin Y. IL-17A contributes to brain ischemia reperfusion injury through calpain-TRPC6 pathway in mice. Neuroscience. (2014) 274:419-28. doi: 10.1016/j.neuroscience.2014.06.001
36. Wang P, Shao BZ, Deng Z, Chen S, Yue Z, Miao CY. Autophagy in ischemic stroke. Prog Neurobiol. (2018) 163-164:98-117. doi: 10.1016/j.pneurobio.2018.01.001

37. Wen YD, Sheng R, Zhang LS, Han R, Zhang X, Zhang XD, et al. Neuronal injury in rat model of permanent focal cerebral ischemia is associated with activation of autophagic and lysosomal pathways. Autophagy. (2008) 4:762-9. doi: $10.4161 /$ auto.6412

38. Carloni S, Girelli S, Scopa C, Buonocore G, Longini M, Balduini W. Activation of autophagy and Akt/CREB signaling play an equivalent role in the neuroprotective effect of rapamycin in neonatal hypoxia-ischemia. Autophagy. (2010) 6:366-77. doi: 10.4161/auto.6.3.11261

39. Kawanokuchi J, Shimizu K, Nitta A, Yamada K, Mizuno T, Takeuchi H, et al. Production and functions of IL-17 in microglia. J Neuroimmunol. (2008) 194:54-61. doi: 10.1016/j.jneuroim.2007.11.006

40. Sutton CE, Mielke LA, Mills KH. IL-17-producing $\gamma$ delta T cells and innate lymphoid cells. Eur J Immunol. (2012) 42:2221-31. doi: 10.1002/eji.2012 42569

41. Lin Y, Zhang JC, Yao CY, Wu Y, Abdelgawad AF, Yao SL, et al. Critical role of astrocytic interleukin-17 A in post-stroke survival and neuronal differentiation of neural precursor cells in adult mice. Cell Death Dis. (2016) 7:e2273. doi: 10.1038/cddis.2015.284

42. Klionsky DJ, Abdelmohsen K, Abe A, Abedin MJ, Abeliovich H, Acevedo Arozena A, et al. Guidelines for the use and interpretation of assays for monitoring autophagy. (3rd edition). Autophagy. (2016) 12:1-222. doi: $10.1080 / 15548627.2015 .1100356$

43. Roux PP, Shahbazian D, Vu H, Holz MK, Cohen MS, Taunton J, et al. RAS/ERK signaling promotes site-specific ribosomal protein S6 phosphorylation via RSK and stimulates cap-dependent translation. J Biol Chem. (2007) 282:14056-64. doi: 10.1074/jbc.M700 906200

44. Biever A, Valjent E, Puighermanal E. Ribosomal protein S6 phosphorylation in the nervous system: from regulation to function. Front Mol Neurosci. (2015) 8:75. doi: $10.3389 /$ fnmol.2015.00075

45. Nygren PJ, Mehta S, Schweppe DK, Langeberg LK, Whiting JL, Weisbrod $\mathrm{CR}$, et al. Intrinsic disorder within AKAP79 fine-tunes anchored phosphatase activity toward substrates and drug sensitivity. Elife. (2017) 6:5653234. doi: 10.7554/eLife.30872.022

Conflict of Interest: The authors declare that the research was conducted in the absence of any commercial or financial relationships that could be construed as a potential conflict of interest.

Copyright $\odot 2019 \mathrm{Liu}, \mathrm{Han}, \mathrm{Dai}$, Zheng, Liu, Li and Li. This is an open-access article distributed under the terms of the Creative Commons Attribution License (CC $B Y)$. The use, distribution or reproduction in other forums is permitted, provided the original author(s) and the copyright owner(s) are credited and that the original publication in this journal is cited, in accordance with accepted academic practice. No use, distribution or reproduction is permitted which does not comply with these terms. 